\title{
Optimal geometric motion planning for spin-stabilized
}

\section{spacecraft}

\author{
James D. Biggs ${ }^{*}$ Nadjim Horri ${ }^{\dagger}$
}

\begin{abstract}
A method requiring low-computational overhead is presented which generates low-torque reference motions between arbitrary orientations for spin-stabilized spacecraft. The initial stage solves a constrained optimal control problem deriving analytical solutions for a class of smooth and feasible reference motions. Specifically, for a quadratic cost function an application of Pontryagin's maximum principle leads to a completely integrable Hamiltonian system that is, exactly solvable in closed-form, expressed in terms of several free parameters. This is shown to reduce the complexity of a practical motion planning problem from a constrained functional optimization problem to an unconstrained parameter optimization problem. The generated reference motions are then tracked using an augmented quaternion feedback law, consisting of the sum of a proportional plus derivative term and a term to compensate nonlinear dynamics. The method is illustrated with an application to re-point a spin-stabilized agile micro-spacecraft using zero propellant. The low computational overhead of the method enhances its suitability for on-board motion generation.
\end{abstract}

Keywords: nonholonomic motion planning, parametric optimization, Pontryagin's maximum principle, attitude control, tracking .

\section{Introduction}

Spin stabilization and three-axis stabilization are methods used to maintain the pointing direction of spacecraft. With spin stabilization the entire spacecraft rotates around its own pointing axis using the gyroscopic effect. ${ }^{1}$ Ad-

*james.biggs@strath.ac.uk, Advanced Space Concepts Laboratory, Department of Mechanical \& Aerospace Engineering, University of Strathclyde, Glasgow, G1 1XJ, UK.

${ }^{\dagger}$ N.Horri@surrey.ac.uk, Surrey Space Centre, Faculty of Engineering and Physical Sciences, University of Surrey, Guildford, Surrey, GU2 7XH, UK. 
vantages of spin stabilization are that it is a simple and passive way of keeping the spacecraft pointing in a certain direction. Designers of early satellites used spin-stabilization and examples include NASA's Pioneer 10 and 11 spacecraft, the Lunar Prospector, and the Galileo Jupiter orbiter. The dynamics and control of spin stabilized spacecraft, under varying mission assumptions, have been extensively researched, see for example. ${ }^{2-5}$ Such passive control is particularly attractive to limited resource spacecraft which can switch off their active GNC system during passive stabilization and use the battery power to perform operational tasks. However, a disadvantage of passive stabilization is that it cannot be used to accurately re-point the antennas or optical instruments. Therefore, missions that require frequent re-pointing tend to use three-axis stabilization. Three-axis stabilization can be achieved with gas jet actuators or reaction wheels using classical attitude control methods such as proportional plus derivative (PD) type controllers, based on quaternion-feedback for large angle maneuvers ${ }^{6}$ or eigenaxis rotations. ${ }^{7}$

The development of nano and micro-spacecraft (from the $1 \mathrm{~kg}$ Cubesat to the more enhanced micro-spacecraft of around $150 \mathrm{~kg}$ ) are cost effective alternatives to conventional spacecraft that pose new engineering challenges. One such challenge is that their small reaction wheels can generate a very limited torque for active control. For example Surrey Satellite Technology Limited (SSTL) is now developing a reaction wheel with a maximum torque capability of $0.1 \mathrm{Nm}$, for future use on micro-satellites $(100 \mathrm{~kg}-150 \mathrm{~kg})$. Therefore, in order to design propellant free maneuvers for micro-spacecraft the reference motions have to be trackable within $0.1 \mathrm{Nm}$. Once the desired pointing direction has been obtained the spacecraft should use passive control to save battery power. Therefore, for micro spacecraft it is desirable to use spin stabilization to maintain a pointing direction and perform small-torque motions (feasible with reaction wheels) for re-pointing. This type of maneuver could be achieved by "de-spinning" the pointing axis, performing a conventional rest-to-rest eigenaxis rotation and finally "spinning-up" around the pointing axis for passive stabilization. However, this three stage manoeuver is a highly inefficient procedure and in general would not be feasible with small torque.

This paper proposes a method requiring low-computational power for generating low-torque reference motions for spacecraft constrained to spin about a single axis. By closely tracking this reference motion the spacecraft will minimize the "spin-up" or "de-spin" of the pointing axis providing gyroscopic stiffness and using small accumulated torque. The design of such low-torque reference motions subject to constraints are often formulated and solved in the context of constrained optimal control problems. These type of problems generally define the attitude kinematics and the Euler equations as equality constraints, with the performance index a function of control torques and/or time 
subject to prescribed boundary conditions and inequality constraints such as bounding the instantaneous torque. ${ }^{8-19}$ In particular, designing minimum accumulated torque motions using pseudo-spectral direct transcription methods has proved instrumental in saving the International Space Station large amounts of precious propellant. ${ }^{20}$ This method can however be computationally demanding. Other optimization approaches such as the locally optimal Euler Lagrange approach of calculus of variations were used to solve problems such as time-optimal attitude control ${ }^{21}$ and the minimum fuel problem for a fixed time horizon. ${ }^{22}$ These local methods have the added complexity of numerically solving two point boundary value problems onboard the satellite. The Hamilton-Jacobi-Bellman (HJB) approach from dynamic programming is globally stabilizing, but is numerically intractable. Solving it off-line is possible numerically, ${ }^{23}$ but the global optimal solution is only approximated up to a certain order, which cannot be too high for practical implementation. For attitude control applications, these numerical optimization techniques are therefore still traditionally avoided onboard small micro-satellites with limited computational resources, such as those developed by SSTL.

The method in this paper uses geometric control theory ${ }^{24-26}$ to reduce the computational expense of the optimization procedure. Geometric control theory has been used to derive coordinate free necessary and sufficient conditions for attitude controllability using gas jet actuators and momentum exchange devices. Furthermore, these results were used to design coordinate free control laws that stabilize the system around an equilibrium state. ${ }^{27}$ Given the controllability of the system it is then desirable to plan a feasible and if possible optimal motion to track. Motion planning for systems defined on Lie groups, e.g. in this case the Special Orthogonal Group $S O(3)$, has been tackled using parameterizations of the manifold and using averaging to design small re-orientations. ${ }^{28}$ However, this method is inadequate to perform large slew maneuvers and a global approach is required. A coordinate free formulation of an optimal control problem was proposed for the attitude motion planning of a spacecraft in Spindler. ${ }^{29}$ For a trivial case of constant optimal angular velocities exact solutions for the corresponding rotations were derived. However, for time-dependent optimal angular velocities the method $\mathrm{in}^{29}$ reverts to using numerical shooting methods, requiring numerical integration, in order to match the prescribed final orientation. Furthermore, this motion planning problem did not take torque requirement into consideration when planning motions.

In this paper, a method is proposed that can rapidly generate low-torque reference motions with low-computational overhead enabled through the machinery of geometric mechanics and control. Although the application of geometric control theory to solve left-invariant optimal control problems and their reduction to quadratures on 3-D Lie groups is well known ${ }^{24,25}$ the particular problem considered here is solvable in closed form. This approach has the advantage 
that,for a spacecraft constrained to spin about one axis, the necessary conditions for optimality are guaranteed exactly with respect to a quadratic cost function. The analytic form of the solution then enables the construction of a practical cost function analytically which, in turn, requires the optimization of only a few free parameters. Specifically, Pontryagin's maximum principle enables the original motion planning problem to be reformulated as an unconstrained parameter optimization problem, thus, greatly reducing the computational demand. Moreover, a numerical optimization of a few parameters is used to match a prescribed final orientation while minimizing torque during the manoeuver. An augmented quaternion feedback is then used to track the designed reference motions.

This paper is presented as follows: In Section II, the kinematic and dynamic models used are introduced. Although the equations for the rigid body are well known they are included here as the kinematics are expressed as quaternions and equivalently in the less conventional matrix form on the Special Unitary Group $S U(2)$. The quaternion differential equations are better suited for numerical integration as they do not have singularities and are conventionally used on-board spacecraft. Furthermore, the compact kinematic formulation on $S U(2)$ is also used as they enable the most natural and elegant analytical treatment in the derivation of the optimal motions. In Section III, the derivation of a general form for the optimal motions in terms of several free parameters is given via Pontryagin's maximum principle and Lax pair integration. ${ }^{24-26}$ The method assigns a simple quadratic cost function to the kinematic equations that constrain the rotational motion of the spinning axis about the pointing direction. It is then shown that the optimal angular velocity inputs are simple sinusoidal functions. This result viewed independently does not explicitly offer any new insight into the control problem as sinusoidal control of nonholonomic systems has already been covered extensively. ${ }^{30}$ However, as the sinusoidal velocity inputs are derived in the setting of geometric mechanics and control it allows the corresponding motion to be derived completely analytically using Lax Pair integration. ${ }^{24,25}$ The derived closed-form analytic expressions then enable the rapid generation of feasible reference motions. In Section IV, an unconstrained numerical parameter optimization method is undertaken to optimize the available parameters of the analytic solution to match the prescribed end-points and minimize accumulated torque amongst this subset of admissible motions.

In Section V, a method is proposed to track the generated reference motions and a proof of the closed-loop stability is given in the appendix. Finally, an example is given which shows the reference motions to be feasible by the tracking controller, which is applied to perform re-pointing manoeuvres for a spin-stabilized agile earth observation SSTL micro-satellite. 


\section{Models}

The equations describing the attitude control problem are that of an asymmetric rigid body with external forces describing the effect of the reaction wheel torques. These consist of kinematic equations relating the angular position to the angular velocity, and the dynamic equations describing the evolution of angular velocity or, equivalently, angular momentum.

\section{A. Kinematic equations}

To obtain a global description of the problem the angular position is usually denoted by a rotation matrix in the Special Orthogonal Group $S O(3)$. In addition the angular position maybe described locally by parameterizing the rotation matrix using Euler angles. ${ }^{27}$ However, in this paper both a quaternion representation and an equivalent matrix formulation on the Special Unitary Group $S U(2)$ is used $\left(S U(2)\right.$ is isomorphic to the unit quaternions $\left.{ }^{25}\right)$. Again this representation is used as the quaternion differential equations are conventionally used on board spacecraft and are suited to numerical integration as they do not possess singularities. In addition the formulation on $S U(2)$ is used as it is the most natural and convenient for the analytical calculations. In terms of the angular velocity $\Omega_{1}, \Omega_{2}, \Omega_{3}$ in the body frame the quaternion representation of kinematics are taken to be:

$$
\frac{d \bar{q}}{d t}=\frac{1}{2} \hat{\Omega} \bar{q}
$$

where $\bar{q}=\left[q_{0}, q\right]^{T}=\left[q_{0}, q_{1}, q_{2}, q_{3}\right]^{T}$ with $q_{0}, q_{1}, q_{2}, q_{3} \in \mathbb{R}$ or equivalently expressed as $\bar{q}=q_{0} \mathbf{e}+q_{1} \mathbf{i}+q_{2} \mathbf{j}+q_{3} \mathbf{k}$ is the unit quaternion where $\mathbf{e}$ is a four-dimensional unit vector and where its orthogonal compliment $\mathbf{i}, \mathbf{j}, \mathbf{k}$ form a right-handed orthonormal frame, $q=\left[q_{1}, q_{2}, q_{3}\right]^{T}$ is known as the vector part of the quaternion and fully determines the attitude where:

$$
\hat{\Omega}=\left(\begin{array}{cccc}
0 & -\Omega_{1} & -\Omega_{2} & -\Omega_{3} \\
\Omega_{1} & 0 & \Omega_{3} & -\Omega_{2} \\
\Omega_{2} & -\Omega_{3} & 0 & \Omega_{1} \\
\Omega_{3} & \Omega_{2} & -\Omega_{1} & 0
\end{array}\right)
$$

The scalar part of the quaternion adds a deliberate redundancy in the attitude parametrization to avoid possible 
attitude propagation singularities. This representation is equivalent to the kinematic matrix representation on $S U(2)$ :

$$
\frac{d R(t)}{d t}=R(t)\left(\Omega_{1} A_{1}+\Omega_{2} A_{2}+\Omega_{3} A_{3}\right)
$$

where $R(t) \in S U(2)$ represents the spacecrafts orientation, $A_{1}, A_{2}, A_{3}$ form a basis for the Lie algebra $\mathfrak{s u}(2)$ of the Lie group $S U(2)$ :

$$
A_{1}=\frac{1}{2}\left(\begin{array}{cc}
i & 0 \\
0 & -i
\end{array}\right), A_{2}=\frac{1}{2}\left(\begin{array}{cc}
0 & 1 \\
-1 & 0
\end{array}\right), A_{3}=\frac{1}{2}\left(\begin{array}{cc}
0 & i \\
i & 0
\end{array}\right)
$$

where $i$ is an imaginary number and the Lie algebra's commutator defined by $[X, Y]=Y X-X Y$ called the Lie bracket with $X, Y \in \mathfrak{s u}(2)$ such that $\left[A_{1}, A_{2}\right]=-A_{3},\left[A_{2}, A_{3}\right]=-A_{1}$ and $\left[A_{1}, A_{3}\right]=A_{2}$ where $R(t) \in S U(2)$ is of the form:

$$
R(t)=\left(\begin{array}{cc}
z_{1} & z_{2} \\
-\bar{z}_{2} & \bar{z}_{1}
\end{array}\right)
$$

with $z_{1}, z_{2} \in \mathbb{C}$ and $\bar{z}_{1}, \bar{z}_{2}$ their complex conjugates such that $\left|z_{1}\right|^{2}+\left|z_{2}\right|^{2}=1$. Physically the basis $A_{1}, A_{2}, A_{3}$ describe the infinitesimal motion of the spacecraft in the roll, pitch and yaw directions respectively. Furthermore, the two sets of kinematic equations (1) and (3) are equivalent with the isomorphism $F: S U(2) \leftrightarrow \mathrm{H}$ :

$$
F:\left(\begin{array}{cc}
z_{1} & z_{2} \\
-\bar{z}_{2} & \bar{z}_{1}
\end{array}\right) \leftrightarrow \mathbf{z}_{1}+\mathbf{z}_{2} \cdot \mathbf{j}=q_{0} \mathbf{e}+q_{1} \mathbf{i}+q_{2} \mathbf{j}+q_{3} \mathbf{k}
$$

defining the coordinate change and where the complex numbers $z_{1}=q_{0}+i q_{1}, z_{2}=q_{2}+i q_{3}$ are regarded in their quaternion form $\mathbf{z}_{1}=q_{0} \mathbf{e}+q_{1} \mathbf{i}, \mathbf{z}_{2}=q_{2} \mathbf{e}+q_{3} \mathbf{i}$ subject to the usual quaternionic multiplication. For more details of this isomorphism see ${ }^{25}$ pp. $169-171$.

\section{B. Dynamic equations}

The Euler equations with external forces are used to represent the attitude dynamics of a spacecraft with reaction wheel actuators:

$$
\begin{array}{r}
\dot{\Omega}_{1}=\delta_{1} \Omega_{2} \Omega_{3}+\frac{u_{1}}{I_{1}} \\
I \dot{\Omega}=-\Omega \times I \Omega+\mathbf{u} \Leftrightarrow \quad \dot{\Omega}_{2}=\delta_{2} \Omega_{1} \Omega_{3}+\frac{u_{2}}{I_{2}} \\
\dot{\Omega}_{3}=\delta_{3} \Omega_{1} \Omega_{2}+\frac{u_{3}}{I_{3}}
\end{array}
$$

where $\delta_{1}=\frac{I_{2}-I_{3}}{I_{1}}, \quad \delta_{2}=\frac{I_{3}-I_{1}}{I_{2}}, \quad \delta_{3}=\frac{I_{1}-I_{2}}{I_{3}}$ where $I_{1}, I_{2}, I_{3}$ are the principal moments of inertia and $I$ is the moment of inertia matrix. The control torque input $\mathbf{u}=\left[u_{1}, u_{2}, u_{3}\right]^{T}$ is generated by reaction wheels aligned with the principal 
axes of the satellite and is given by $\mathbf{u}=-\dot{\mathbf{h}}-\Omega \times \mathbf{h}$ where $\Omega=\left[\begin{array}{lll}\Omega_{1}, & \Omega_{2}, & \Omega_{3}\end{array}\right]^{T}$ and the vector $\mathbf{h}$ represents the angular momentum of the wheels.

\section{Analytic derivation of the reference motion}

The initial formulation of the motion planning problem is presented in the context of a constrained functional optimization problem that includes equality constraints and a performance index (an integral function of the angular velocities). This formulation ensures smooth and feasible motions are defined and enable the derivation of analytic solutions in closed form. This renders a class of feasible curves subject to the equality constraint that satisfy the necessary conditions for optimality. This analytic form essentially reduces the original constrained functional optimization problem to an unconstrained parameter optimization problem where the parameters are chosen to match the boundary conditions and minimize accumulated torque. The initial motion planning problem is defined by the kinematic constraint (equality constraint):

$$
\frac{d R(t)}{d t}=R(t)\left(v A_{1}+\Omega_{2} A_{2}+\Omega_{3} A_{3}\right)
$$

where the roll axis in constrained to spin at a constant rate $\Omega_{1}=v$. Note that this also includes the possibility of performing maneuvers where the pointing direction is constrained to have zero spin $v=0$. Amongst all admissible motions of (8) we seek solutions that minimize the functional $l(R(t))=\int_{0}^{T}\left\langle\frac{d R(t)}{d t}, \frac{d R(t)}{d t}\right\rangle d t$ between the given boundary conditions $R(0)=R_{0}$ and $R(T)=R_{T}$ where $T$ is a fixed-terminal time and $\langle\cdot, \cdot\rangle=-\frac{1}{2} \operatorname{trace}(\cdot, \cdot)$ is the trace form. As the trace form is left (respectively right) invariant this is equivalent to minimizing $l(R(t))=$

$\int_{0}^{T}\left\langle R(t)^{-1} \frac{d R(t)}{d t}, R(t)^{-1} \frac{d R(t)}{d t}\right\rangle d t$ and from (8) it follows that $l(R(t))=\frac{1}{2} \int_{0}^{T} v^{2}+\Omega_{2}^{2}+\Omega_{3}^{2} d t$. As $v$ is constant on the fixed-time interval $T$ this is equivalent to minimizing the performance index:

$$
f_{0}=\frac{1}{2} \int_{0}^{T} \Omega_{2}^{2}+\Omega_{3}^{2} d t
$$

It is easily shown that this problem is controllable and therefore this optimal control problem is well posed. ${ }^{24,25}$ This initial cost function is not conventional but it is meaningful since (i) it ensures smooth motions (ii) it minimizes the integral of angular velocities on the unconstrained axes which avoids the system accumulating more angular velocity than needed (iii) it avoids dangerously fast slew rates while making sure that the final attitude is specified (not at rest in final time but with small bounded final velocity) and (iv) using the machinery of geometric control theory it allows the construction of the optimal motions in closed form: 
Theorem 1 The class of reference motions that minimize the cost function (9) subject to the equality constraint (8) are defined by:

$$
\begin{aligned}
\Omega_{2}^{*}= & r \sin ((v+c) t+\beta), \quad \Omega_{3}^{*}=r \cos ((v+c) t+\beta) \\
q_{0}^{*} & =\frac{(K-c) \cos \left(\frac{1}{2} t(c-K+v)\right)+(K+c) \cos \left(\frac{1}{2} t(c+K+v)\right)}{2 K} \\
q_{1}^{*} & =\frac{(K-c) \sin \left(\frac{1}{2} t(c-K+v)\right)+(K+c) \sin \left(\frac{1}{2} t(c+K+v)\right)}{2 K} \\
q_{2}^{*} & = \pm \frac{r}{K} \sin \left(\frac{(v+c)}{2} t+\beta\right) \sin \left(\frac{K}{2} t\right) \\
q_{3}^{*} & = \pm \frac{r}{K} \cos \left(\frac{(v+c)}{2} t+\beta\right) \sin \left(\frac{K}{2} t\right)
\end{aligned}
$$

where $\Omega_{1}^{*}=v, \Omega_{2}^{*} \Omega_{3}^{*}$ are the optimal angular velocities and $q_{0}^{*}, q_{1}^{*}, q_{2}^{*}, q_{3}^{*}$ the corresponding quaternion components subject to the given boundary conditions $\bar{q}^{*}(0)=\left[\begin{array}{llll}1 & 0 & 0 & 0\end{array}\right]^{T}$ and $\bar{q}^{*}(T)=\bar{q}_{T}$ and where $r, c, \beta$ are parameters available for optimization, $v$ is the given spinning angular velocity and $K=\sqrt{c^{2}+r^{2}}$ is a constant.

Proof

The Hamiltonian corresponding to the left-invariant kinematic constraint (8) that minimises the function (9) is: ${ }^{24-26}$

$$
H(p, u)=v p\left(A_{1}\right)+\Omega_{2} p\left(A_{2}\right)+\Omega_{3} p\left(A_{3}\right)-\rho_{0}\left(\frac{1}{2}\left(\Omega_{2}^{2}+\Omega_{3}^{2}\right)\right)
$$

where $\rho_{0} \geqslant 0$ is a fixed positive constant and $p(\cdot): \mathfrak{s u}(2) \mapsto \mathbb{R}$ such that $p\left(A_{i}\right)$ are scalar components of an element in the dual of the Lie algebra $\mathfrak{s u}(2)^{*}$, with $\rho_{0}=1$ for regular extremals and $\rho_{0}=0$ for abnormal extremals. It can be shown that the abnormal extremals are projections of the regular extremals so set $\rho_{0}=1$ (see Jurdjevic (1997) ${ }^{25}$ for details). Following this define the linear functions $\lambda_{i}=p\left(A_{i}\right)$ for $i=1,2,3$ then from the Maximum Principle and the fact that (12) is a concave function the optimal velocity inputs are given by $\frac{d H}{d \Omega_{i}}=0$ and therefore $\Omega_{2}^{*}=\lambda_{2}, \Omega_{3}^{*}=\lambda_{3}$ Substituting these values into (12) gives the optimal Hamiltonian

$$
H^{*}=v \lambda_{1}+\frac{1}{2}\left(\lambda_{2}^{2}+\lambda_{3}^{2}\right)
$$

Defining the Poisson Bracket $\left\{\lambda_{i}, \lambda_{j}\right\}=-p\left(\left[A_{i}, A_{j}\right]\right)$ where $\left[A_{i}, A_{j}\right]$ is the Lie bracket then $\dot{\lambda}_{i}=\left\{H, \lambda_{i}\right\}$ is:

$$
\dot{\lambda}_{1}=0, \quad \dot{\lambda}_{2}=\lambda_{3}\left(v-\lambda_{1}\right), \quad \dot{\lambda}_{3}=-\lambda_{2}\left(v-\lambda_{1}\right)
$$

where $\lambda_{1}, \lambda_{2}, \lambda_{3} \in \mathfrak{s u} \mathfrak{u}^{*}(2)$. For convenience we define $\lambda_{1}=-c$ where $c$ is a constant. Defining a reduced constant Hamiltonian $H_{r}=2\left(H^{*}+c v\right)$ gives $H_{r}=\lambda_{2}^{2}+\lambda_{3}^{2}$ then parameterizing $H_{r}$ using polar coordinates $\lambda_{2}=r \sin \theta, \lambda_{3}=$ $r \cos \theta$ gives $r=H_{r}^{1 / 2}$ and $\theta$ is given as follows:

$$
\theta=\arctan \left(\frac{\lambda_{2}}{\lambda_{3}}\right)
$$


then

$$
\dot{\theta}=\frac{\lambda_{3} \dot{\lambda}_{2}-\lambda_{2} \dot{\lambda}_{3}}{\lambda_{2}^{2}+\lambda_{3}^{2}}
$$

substituting $\dot{\lambda}_{2}$ and $\dot{\lambda}_{3}$ from (14) into (16) yields:

$$
\dot{\theta}=v+c
$$

therefore $\theta=C_{1} t+\beta$ where $C_{1}=v+c$ and $\beta$ is a constant of integration. To obtain the corresponding quaternions, we use the Lax pair representation of the regular extremals: ${ }^{25}$

$$
\frac{d L(t)}{d t}=[L(t), \Delta H]
$$

where

$$
L(t)=\lambda_{1} A_{1}+\lambda_{2} A_{2}+\lambda_{3} A_{3}, \quad \Delta H=v A_{1}+\lambda_{2} A_{2}+\lambda_{3} A_{3}
$$

with general solution:

$$
L(t)=R(t)^{-1} L(0) R(t)
$$

equivalently

$$
R(t) L(t) R(t)^{-1}=L(0)
$$

where $L(0)$ is a matrix of constant entries and $R(t) L(t) R(t)^{-1}$ describes the conjugacy class of $L(t)$ and therefore an initial $R(0)$ can be chosen such that $L(0)=K A_{1}$. Therefore, it suffices to integrate the particular solution:

$$
\bar{R}(t) L(t) \bar{R}(t)^{-1}=K A_{1}
$$

therefore

$$
L(t)=K \bar{R}(t)^{-1} A_{1} \bar{R}(t)
$$

as $\exp \left(\varphi_{1} A_{1}\right)$ is the stabilizer of $A_{1}$ it is convenient to introduce the coordinate form:

$$
\bar{R}(t)=\exp \left(\varphi_{1} A_{1}\right) \exp \left(\varphi_{2} A_{2}\right) \exp \left(\varphi_{3} A_{1}\right)
$$

and substituting into (23) yields:

$$
L(t)=\frac{i K}{2}\left(\begin{array}{cc}
\cos \varphi_{2} & e^{-i \varphi_{3}} \sin \varphi_{2} \\
e^{i \varphi_{3}} \sin \varphi_{2} & -\cos \varphi_{2}
\end{array}\right)
$$

then equating (25) with $L(t)$ in (19) yields

$$
\lambda_{1}=K \cos \varphi_{2}, \quad \lambda_{2}+i \lambda_{3}=e^{-i \varphi_{3}} \sin \varphi_{2}, \quad-\lambda_{2}+i \lambda_{3}=e^{i \varphi_{3}} \sin \varphi_{2}
$$


which can also be simplified to obtain:

$$
\tan \varphi_{3}=\frac{\lambda_{2}}{\lambda_{3}}
$$

to obtain an expression for $\varphi_{1}$ substitute (24) into (3) and simplify using the previously derived expressions to yield:

$$
\dot{\varphi}_{1}=K
$$

and define the initial angle to be $\varphi_{1}(0)=\gamma$ which yields $\varphi_{1}=K t+\gamma$. Substituting $\varphi_{2}$ and $\varphi_{3}$ in from equations (26) and (27) into (24) and pulling the solution back to the identity via:

$$
R(t)=R_{\text {int }} \bar{R}(0)^{-1} \bar{R}(t)
$$

where $R_{\text {int }}$ is the initial orientation and $\bar{R}(0)^{-1}$ is the inverse of $\bar{R}(t)$ at $t=0$ gives the solution on $S U(2)$. Finally, using the isomorphism (6) yields (11). Q.E.D.

This defines analytically a subset of admissible smooth motions expressed in terms of several free parameters. Note that the sign of $q_{2}, q_{3}$ in (11) is dependent on choosing the frame to be positively or negatively oriented. For this analytic solution to correspond with the numerical solution of the differential equation (1) this frame is taken to be negatively oriented. The following section assigns analytically derived cost functions (also functions of the same free parameters) which can be numerically optimized to minimize the accumulated torque and prescribed final orientation error. This initial constrained optimal control problem defined amongst all admissible curves has been reduced to a class of analytically defined feasible curves. This essentially reduces the motion planning problem to an unconstrained parameter optimization problem.

\section{Unconstrained parameter optimization}

The previous section derives an analytic description of a class of smooth, feasible motions expressed as functions of the parameters $v, r, c, \beta(10,11)$. The problem now is to choose the free parameters $r, c, \beta$ such that the boundary conditions are matched at the terminal time $t=T$ (they are not included in the original performance index) and such that accumulated torque is minimized. In order to match a prescribed final pointing direction $\bar{q}_{f}=\left[\begin{array}{llll}q_{f 0} & q_{f 1} & q_{f 2} & q_{f 3}\end{array}\right]^{T}$ at the terminal time $t=T$ to high-precision the available parameters can be optimized to minimize the performance index:

$$
J_{1}=\left[\left(q_{0}-q_{f 0}\right)^{2}+\left(q_{1}-q_{f 1}\right)^{2}+\left(q_{2}-q_{f 2}\right)^{2}+\left(q_{3}-q_{f 3}\right)^{2}\right]_{t=T}
$$


In addition, when planning a motion for micro-spacecraft it is essential to minimize torque during the manoeuver. A cost functional that penalizes torque requirement can be expressed in terms of these free parameters. Substituting (10) and $\Omega_{1}=v$ into (7) yields the control torque input $\mathbf{u}=\left[\begin{array}{lll}u_{1}, & u_{2}, & u_{3}\end{array}\right]^{T}$ :

$$
\begin{aligned}
& u_{1}=-(1 / 2) I_{1} r^{2} \sin (2(t(c+v)+\beta)) \delta_{1} \\
& u_{2}=I_{2} r \cos (t(c+v)+\beta)\left(c+v-v \delta_{2}\right) \\
& u_{3}=-I_{3} r \sin (t(c+v)+\beta)\left(c+v+v \delta_{3}\right)
\end{aligned}
$$

Then to minimize the accumulated torque over the time interval $t \in[0, T]$ the available parameters $r, c, \beta$ can be optimized such that the functional:

$$
J_{2}=\int_{0}^{T}\langle\mathbf{u}, \mathbf{u}\rangle^{2} d t
$$

is minimized. This integral can be evaluated analytically and equates to finding the minimum of the analytic function:

$$
J_{2}=\frac{r^{2}}{32 \alpha}\left(\begin{array}{c}
r^{2}(4 T \alpha+\sin (4 \beta))-\sin (4(T \alpha+\beta)) I_{1}^{2} \delta_{1}^{2} \\
+8\left(2 T \alpha-\sin (2 \beta)+\sin (2(T \alpha+\beta)) I_{2}^{2}\left(\alpha+v \delta_{2}\right)^{2}\right. \\
+8\left(2 T \alpha+\sin (2 \beta)-\sin (2(T \alpha+\beta)) I_{3}^{2}\left(\alpha+v \delta_{3}\right)^{2}\right.
\end{array}\right)
$$

where $\alpha=v+c$. Therefore, to obtain the optimal parameters for the reference motion $(10,11)$ the multi-objective function:

$$
J=J_{1}+k J_{2}
$$

is minimized, where $J_{1}$ and $J_{2}$ are defined in (30) and (33) respectively and where $k$ is a weighting parameter. Given the moments of inertia $I_{1}, I_{2}, I_{3}$, spinning angular velocity $v$ and terminal time $T$ this problem is easily solved using unconstrained numerical parameter optimization.

\section{Re-pointing an agile spin stabilized micro-spacecraft}

In this section the methodology is applied to perform a large slew-manoeuver for a spin-stabilized spacecraft. The model of an agile earth observation micro-satellite (based on a small satellite platform from Surrey Satellite Technology Limited (SSTL)) is used for the numerical simulation study. The moments of inertia of the micro-satellite are given by $I_{1}=19.5 \mathrm{kgm}^{2}, I_{2}=19 \mathrm{kgm}^{2}, I_{3}=12.6 \mathrm{kgm}^{2}$. The less significant cross terms of the moment of inertia matrix are neglected for simplicity. A feasible spinning velocity constraint of $v=0.2 \operatorname{rad} s^{-1}$ is assumed.

Furthermore, it is desired to perform a large slew manoeuver from $q_{0}=\left[\begin{array}{llll}1 & 0 & 0 & 0\end{array}\right]^{T}$ to $q_{f}=\left[\begin{array}{llll}0 & 0 & 1 & 0\end{array}\right]^{T}$ in $T=$ 
200 secs (a reasonable time-scale for such a manoeuver to be performed with admissible torque). Two unconstrained parameter optimizations of the performance index (34) were undertake for comparison using Mathematica: ${ }^{31}$ (i) when $k=0$ and (ii) when $k=1 / 10$. When $k=0$, the numerical parameter optimization returns the parameter values $r=0.6754, c=-3.6494 \times 10^{-8}, \beta=-56.1358$. Substituting these values into (30) and (33) yields $J_{1}=2.1982 \times 10^{-7}$ and $J_{2}=5.5684$ respectively. By comparison, when $k=1 / 10$ an unconstrained numerical optimization yields the parameters $r=0.0157, c=-7.1920 \times 10^{-8}, \beta=0.4204$. Substituting these values into (30) and (33) yields $J_{1}=$ $4.2081 \times 10^{-6}$ and $J_{2}=0.0026$. Comparing the two results it can be seen that there is an inevitable trade off between final orientation error and accumulated torque depending on the weight $k$. However, there is only a small increase in final orientation error when compared to the decrease in accumulated torque. Moreover, when $k=0$ it was found that the resulting reference motion could not be tracked effectively with micro-reaction wheels (maximum torque of 0.1 $\mathrm{Nm}$ ). However, as is now shown the reference motion generated for $k=1 / 10$ is trackable using micro-reaction wheels. Substituting the generated parameters $r=0.0157, c=-7.1920 \times 10^{-8}, \beta=0.4204$ into (10) yields the reference angular velocities:

$$
\begin{aligned}
& \Omega_{1}^{*}=0.2 \mathrm{rads}^{-1} \\
& \Omega_{2}^{*}=0.015708 \sin (0.420394+0.2 t) \mathrm{rads}^{-1} \\
& \Omega_{3}^{*}=0.015708 \cos (0.420394+0.2 t) \mathrm{rads}^{-1}
\end{aligned}
$$

and substituting these parameters into (11) yields the corresponding reference quaternion components:

$$
\begin{aligned}
& q_{0}^{*}=0.5 \cos (0.092146 t)+0.5 \cos (0.107854 t) \\
& q_{1}^{*}=0.5 \sin (0.092146 t)+0.5 \sin (0.107854 t) \\
& q_{2}^{*}=-\sin (0.4204+0.1 t) \sin (0.00785398 t) \\
& q_{3}^{*}=-\cos (0.4204+0.1 t) \sin (0.00785398 t)
\end{aligned}
$$

finally substituting the generated parameters into (31) gives the theoretical torque history required to track this reference motion:

$$
\begin{aligned}
& u_{1}=-0.000789567 \sin (2(0.420359+0.2 t)) \mathrm{Nm} \\
& u_{2}=0.0813672 \cos (0.420359+0.2 t) \mathrm{Nm} \\
& u_{3}=-0.0411548 \sin (0.420359+0.2 t) \mathrm{Nm}
\end{aligned}
$$

Following a transient tracking stage which will depend on controller gains and initial conditions, the actual torque required to track this trajectory, shown in Figure 1, approaches the trigonometric expressions of the torque given in 
equation (37). The tracking of this reference motion is performed by the augmented quaternion feedback controller: ${ }^{6}$

$$
\mathbf{u}=-\dot{\mathbf{h}}-\Omega \times \mathbf{h}=-k_{p} I q_{e}-k_{d} I \Omega_{e}+\Omega \times I \Omega+I \dot{\Omega}^{*}
$$

where $k_{p}$ and $k_{d}$ are scalar gains being multiplied by the moment of inertia matrix $I$ to make sure that gains are higher on the axes with a higher moment of inertia. The vectors $q_{e}$ and $\Omega_{e}$ denote the vector part of the quaternion tracking error and the angular velocity tracking error vector respectively.

Note that the variable $\mathbf{u}$ was introduced to simplify the stability proof in the appendix. The first two terms of the control law (38) are used to tune the convergence rate and to adjust the damping characteristic, which varies nonlinearly. The third term is a gyroscopic coupling nonlinearity and the last term is added to compensate for the acceleration errors that would otherwise result from the time variability of the optimal angular velocities. The proposed tracking approach, based on PD quaternion feedback with compensation of nonlinearities, is chosen as it has been flight proven for other control objectives with time varying trajectories such as Sun tracking. It is also adequate for the tracking of smooth trajectories, guarantees stability and only requires the tuning of two gains.

In practice, the control input is the control torque vector of the wheels $-\dot{\mathbf{h}}$, which is easily obtained using the fact that $-\dot{\mathbf{h}}=\mathbf{u}+\Omega \times \mathbf{h}$. The wheels momenta $h_{i}, i=1,3$ can then be integrated from an initial value and the wheels speed commands are easily obtained by division about the wheels moments of inertia $I_{w h i}$. The quaternion error is obtained using the quaternion composition rule. More explicitly, the angular velocity error and quaternion error are respectively given by:

$$
\Omega_{e}=\Omega-\Omega^{*}, \quad \bar{q}_{e}=Q^{*} \bar{q}
$$

where $Q^{*}=\left[\begin{array}{rrrr}q_{4}^{*} & q_{3}^{*} & -q_{2}^{*} & -q_{1}^{*} \\ -q_{3}^{*} & q_{4}^{*} & q_{1}^{*} & -q_{2}^{*} \\ q_{2}^{*} & -q_{1}^{*} & q_{4}^{*} & -q_{3}^{*} \\ q_{1}^{*} & q_{2}^{*} & q_{3}^{*} & q_{4}^{*}\end{array}\right]$ is defined from the quaternion command vector $\bar{q}^{*}=\left[q_{0}^{*}, q_{1}^{*}, q_{2}^{*}, q_{3}^{*}\right]$ from equation (36).

The differential equation for the angular velocity error can be trivially obtained from equation (39). The differential equation for the quaternion error $\bar{q}_{e}$ has an analogous form to the one for the actual quaternion $\bar{q}$ :

$$
\frac{d \bar{q}_{e}}{d t}=\frac{1}{2} \hat{\Omega}_{e} \bar{q}_{e}
$$


where $\hat{\Omega}_{e}$ denotes the matrix form of the angular velocity error of the form (2), $\bar{q}_{e}=\left[q_{e 0}, q_{e}^{T}\right]^{T}=\left[q_{e 0}, q_{e 1}, q_{e 2}, q_{e 3}\right]^{T}$ and the equation (40) is similar to equation (1) by taking the components of $\bar{q}_{e}$ rather than those of $\bar{q}$.

The stability proof is provided in the appendix. The controller tuning is performed experimentally through extensive numerical simulation. In the literature, there is an algorithmic approach for tuning the gain in the special case of small eigenaxis rotations satisfying $\Omega=\dot{\theta} e$ and $q=\sin (\theta / 2) e$. Under the assumption of rest-to-rest maneuvers, the closed loop system reduces to a second order oscillator ${ }^{6}$ which can be used to determine the gain. Although this paper deals with global, large slew-motions the use of this procedure $^{6}$ was used to get a first estimate of the gains. In addition the procedure of the trial and error simulations are made simpler here by making the gain matrices proportional to the moment of inertia matrix, meaning that only two gains need to be determined, despite this being a three axis control problem.

A torque saturation limit of $0.1 \mathrm{Nm}$ is considered in this simulation study. A sampling time of 1 second is used, that is, all measurements of attitude, angular velocity and control torque are available every second. Furthermore, zero order holds of 1 second are included in the model which essentially keep the continuous variables constant for 1 second. Initial angular velocity and quaternion errors were introduced to illustrate that the controller does not require a transient velocity control stage before activation.

After extensive numerical simulations of the resulting closed loop dynamics (in nonzero momentum mode), three sets of controller parameters were chosen, in three separate simulations, to illustrate the effect of controller parameters on tracking performance and torque expenditure:

$$
\begin{aligned}
& \text { Low tracking gains: } k_{p}=0.025, \quad k_{d}=0.16 \\
& \text { Medium tracking gains: } k_{p}=0.06, \quad k_{d}=0.48 \\
& \text { High tracking gains: } k_{p}=0.5, \quad k_{d}=4.5
\end{aligned}
$$

Note that these gains were chosen to compare tracking performance for similar damping characteristics. Increasing the derivative gains further on the axes with time varying desired velocities was found not to necessarily increase damping on all axes because of the system's nonlinearity.

For a final boundary condition at 200 seconds, Figure 1 shows the control torque required to track the optimal angular velocity trajectories with the high set of gains $k_{p}=0.5, k_{d}=4.5$, while Figure 2 shows the same plot for low gains $k_{p}=0.025, k_{d}=0.16$. The simulation shows that the control torque is feasible using $0.1 \mathrm{Nm}$ wheels with the lower set of gains, but not with the higher one. Note that the analytically computed control torques of equation 


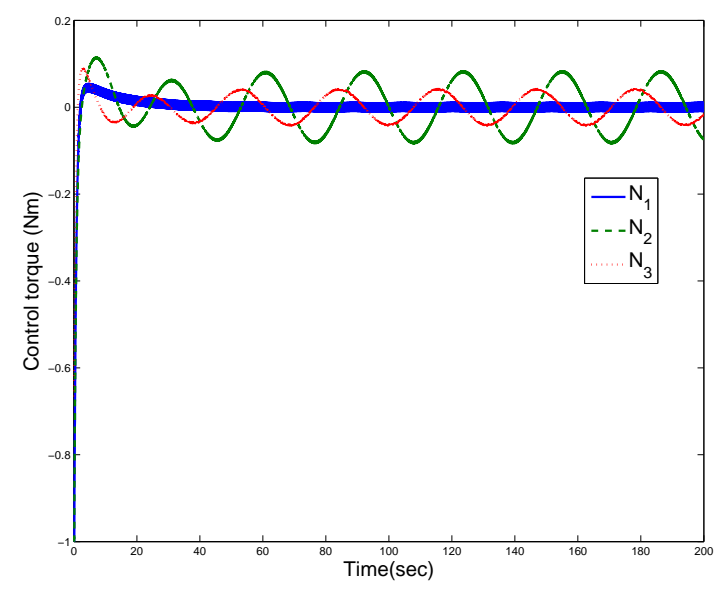

Figure 1. Control torque of the reaction wheels- High gains

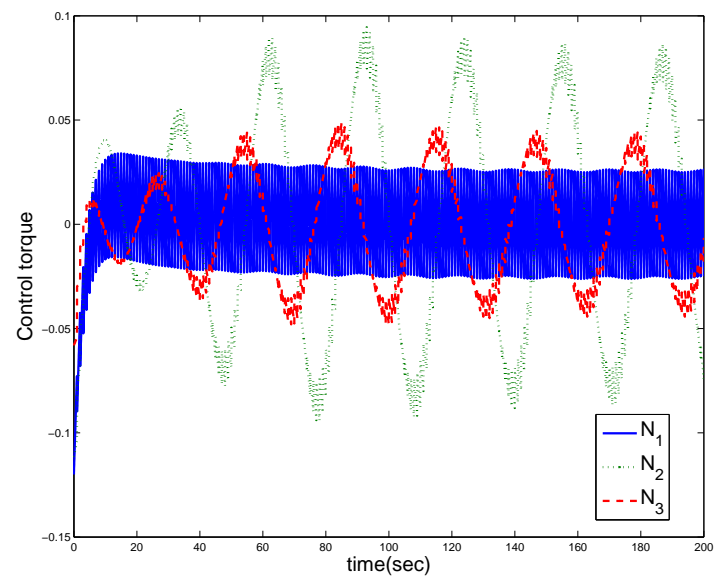

Figure 2. Control torque of the reaction wheels- Low gains

(37) are approached closely at steady state after transient errors. Figure 3 shows that the angular velocity tracking is enhanced at steady state, but not at the transient time, by increasing the gains. Figure 4 shows that the attitude tracking performance is enhanced on all three axes at steady states, but not at the transient time, by increasing the PD gains. Figure 5 also shows a similar trend.

Note that although the results show that the torque requirement for good tracking is feasible for micro-spacecraft, the required torque capability can be reduced by increasing the time horizon of the unconstrained numerical parameter optimization procedure at the reference motion design stage. 

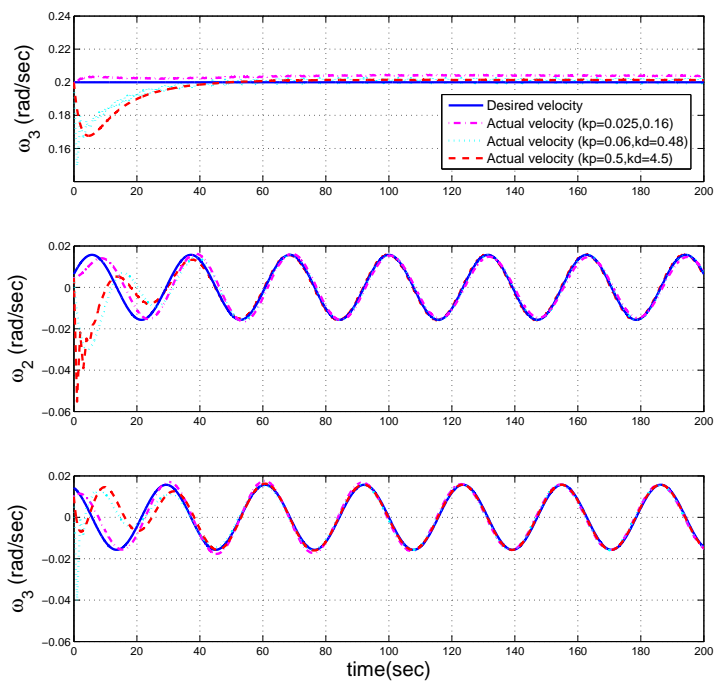

Figure 3. Tracking of the desired angular velocity
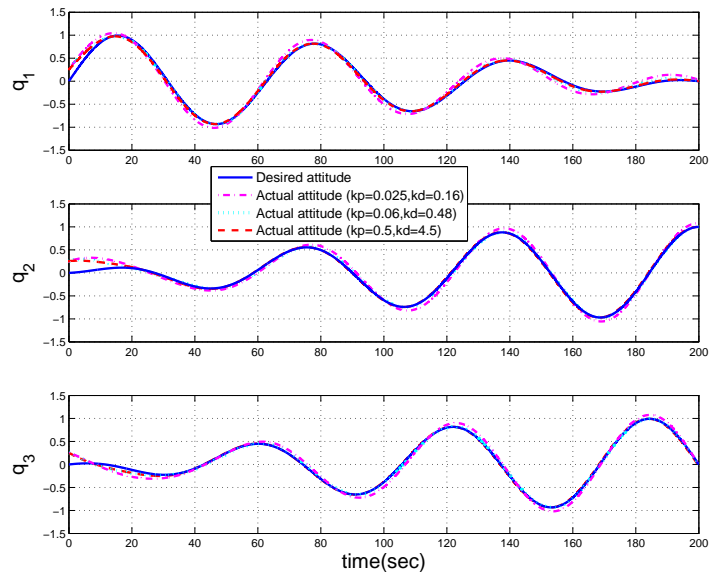

Figure 4. Tracking of the desired attitude

\section{Conclusion}

This paper has presented a method for generating low-torque attitude reference motions requiring low-computational overhead for spin-stabilized spacecraft. It was further illustrated through simulation that these motions can be tracked with admissible torque using reaction wheels onboard a micro-satellite. The procedure used for the motion planning involved reducing the initial constrained functional optimization problem to an unconstrained parameter optimization 

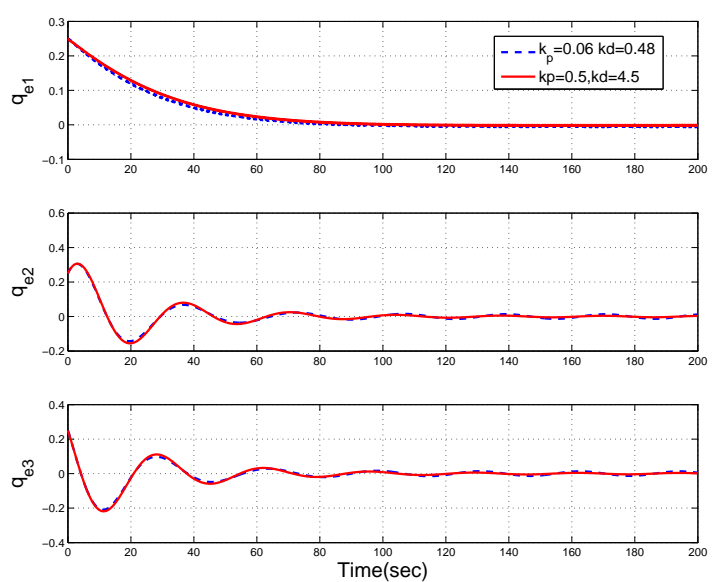

Figure 5. Quaternion errors

problem via Pontryagin's maximum principle. A numerical parameter optimization was then employed to minimize the final orientation error, between the actual and desired final orientation, and the accumulated torque during the motion. One of the main advantages of this approach is that it requires the optimization of only a few parameters and thus is able to rapidly generate low-torque reference motions using low-computational power. It was further shown that the reference motions can be tracked by limited resource spacecraft using an augmented version of the conventional, flight tested, quaternion feedback law. In particular, it was illustrated that a generated large slew reference motion was feasible for a near term micro-spacecraft with admissible torque and good tracking performance.

\section{Appendix}

The stability of the system with the controller of equation (38) can be shown with respect to the following Lyapunov function:

$$
V=k_{p}\left(q_{e 1}^{2}+q_{e 2}^{2}+q_{e 3}^{2}+\left(1-q_{e 4}\right)^{2}\right)+\frac{1}{2} \Omega_{e}^{T} \Omega_{e}
$$

The first term can be simplified using the norm conservation property of the quaternion:

$$
V=2 k_{p}\left(1-q_{e 4}\right)+\frac{1}{2} \Omega_{e}^{T} \Omega_{e}
$$

The differentiation of $V$ leads to :

$$
\dot{V}=-2 k_{p} \dot{q}_{e 4}+\Omega_{e}^{T} \dot{\Omega}_{e}
$$


By substituting $\mathbf{u}$ by its expression from equation (38), we have:

$$
\dot{V}=k_{p} \Omega_{e}^{T} q_{e}+\Omega_{e}^{T} I^{-1}\left(-k_{p} I q_{e}-k_{d} I \Omega_{e}+I \dot{\Omega}^{*}-I \dot{\Omega}^{*}\right)
$$

A number of terms in the last equation cancel and the resulting time derivative of $V$ reduces to:

$$
\dot{V}=-k_{d} \Omega_{e}^{T} \Omega_{e}<0 \quad \forall \Omega_{e} \neq 0
$$

At the equilibrium point, $V\left(\bar{q}_{e}=[1,0,0,0]^{T}, \Omega_{e}=[0,0,0]^{T}\right)=0$, we have $V=0$. Convergence to the equilibrium follows from Lasalle's invariance principle. ${ }^{33}$

PD feedback with compensation of nonlinearities is known to be robust in practice to the bounded disturbances that are linearly related to attitude dynamics and nearly periodic functions of time. For applications requiring robustness under arbitrary bounded disturbance torques, an alternative would be to substitute the PD terms by a sliding mode term or to simply add a sliding mode term to robustify the stabilizing controller, as proposed by Luo et al. ${ }^{32}$

\section{References}

${ }^{1}$ Sidi, M. J., 'Spacecraft Dynamics and Control: A practical engineering approach ', Cambridge University Press, 2002.

${ }^{2}$ Hall C, D., Rand. R, H., 'Spinup Dynamics of Axial Dual-Spin Spacecraft', Journal of Guidance, Control and Dynamics, Vol. 17, No. 1, pp. $30: 37,1994$

${ }^{3}$ Meehan, P. A., Asokanthan, S. F., 'Control of Chaotic Motion in a Dual-Spin Spacecraft with Nutational Damping', Journal of Guidance, Control and Dynamics, Vol. 25, No. 2, pp. 209:214, 2002.

${ }^{4}$ Bao, G. W., Pascal, M., 'Stability of a Spinning Liquid-filled Spacecraft, Applied Mechanics, Vol. 67, pp. 407:421, 1997.

${ }^{5}$ Tsiotras, P., Longuski, J. M., 'Spin-axis Stabilization of Symmetric Spacecraft with Two Control Torques', Systems and Control Letters, Vol. 23, pp. 395:402, 1994.

${ }^{6}$ Wie, B., Barba, P. M. 'Quaternion feedback for spacecraft large-angle maneuvers'. Journal of Guidance, Control and Dynamics, vol. 8, No. 3, pp. 360-365, 1985.

${ }^{7}$ Wie, B., Weiss, H.,Arapostathis, A. 'Quaternion feedback regulator for spacecraft eigenaxis rotations'. Journal of Guidance, 12,375380 , 1989.

${ }^{8}$ Scrivener, S. L., Thompson, R. C. 'Survey of Time-Optimal attitude Maneuvers'. Journal of Guidance, Control, and Dynamics, Vol. 17, No. 2, pp. 225-231, 1994.

${ }^{9}$ Vadali, S.R., Junkins, J.L. 'Optimal open loop and stable feedback control of rigid spacecraft attitude maneuvers', Journal of Astronautical sciences, Vol 32, no2,pp. 105-122, april-june 1984. 
${ }^{10}$ Junkins,J. L., Carrington, C. K., and Williams, C. E., 'Time-optimal magnetic attitude maneuvers,' J. Guidance, Control, and Dynamics, vol. 4, no. 4, pp. 363368, 1981.

${ }^{11}$ Byers, R. M., and Vadali, S. R. 'Quasi closed-form solution to the time-optimal rigid spacecraft reorientation problem,' AAS Paper 91120 , 1991.

${ }^{12}$ Etter, J. R., 'A solution of the time-optimal Euler rotation problem,' AIAA Paper 89-3601-CP, 1989.

${ }^{13}$ Tsiotras, P., 'Stabilization and Optimality Results for the Attitude Control Problem’ Journal of Guidance Control and Dynamics Vol.19, No. 4, July-August 1996.

${ }^{14}$ Athans, M. , Falb, P. L., and Lacoss, R. T., 'Time-, fuel-, and energy optimal control of nonlinear norm-invariant systems,' IRE Trans. Automat. Contr., vol. 8, pp. 196202, July 1963.

${ }^{15}$ Dixon, M. V., Edelbaum, T., Potter, J. E., and Vandervelde, W. E., 'Fuel optimal reorientation of axisymmetric spacecraft,' J. Spacecraft, vol. 7, pp. 13451351,1970

${ }^{16}$ Wolske, G. D., 'Minimum fuel attitude control of non-linear satellite system’, Int. J. Non-Linear Mechanics, vol. 5, pp. 557-575, 1970.

${ }^{17}$ Boyarko, G. A., Romano, M. Yakimenko, O. A., 'Minimum fuel control of high-order systems', Journal of optimisation theory and applications, vol. 48, No. 3, pp. 469-491, 1986.

${ }^{18}$ Feiyue Li, Peter Bainum, 'Numerical approach for solving rigid spacecraft minimum time attitude maneuvers', AIAA Journal of Guidance, Control and Dynamics, Vol 13, no1, pp.38-45, 1990.

${ }^{19}$ Boyarko, G.A., Romano, M., Yakimenko, O.A. 'Time-optimal reorientation of a spacecraft using a direct optimisation method based on inverse dynamics', IEEE Aerospace Conference, Big Sky, Montana, USA, 2010.

${ }^{20}$ Kang, W., Bedrossian, N., 'Pseudospectral optimal control theory makes debut flight, saves NASA $\$ 1 \mathrm{M}$ un under three hours', SIAM News, Vol. 40, No. 7, 2007.

${ }^{21}$ Bilimoria, K.D., Wie, B., 'Time-optimal three-axis reorientation of a rigid spacecraft'. AIAA Journal of Guidance, Control, and Dynamics, vol. 16 , no. 3 , pp. 446-452, 1993.

${ }^{22}$ Seywald, H., Kumar, R.R., Deshpande, S.S., Heck, M.L. 'Minimum fuel spacecraft reoriantation'. AIAA Journal of Guidance, Control, and Dynamics, vol. 17, no. 1, pp. 21-29, 1994.

${ }^{23}$ Lawton, J., Beard, R., Mclain, T. 'Successive Galerkin approximation of nonlinear optimal attitude control'. Proceedings of the American Control Conference, San Diego, California, June, 1999.

${ }^{24}$ Jurdjevic, V., 'Integrable Hamiltonian Systems on Complex Lie Groups'. Memoirs of the American Mathematical Society, No. 838, vol. 178, 2005.

${ }^{25}$ Jurdjevic, V., ‘Geometric Control Theory’. Advanced Studies in Mathematics, Cambridge University Press, 52, 1997.

${ }^{26}$ Sussmann, H. J., 'An introduction to the coordinate-free maximum principle'. In Geometry of Feedback and Optimal Control,B. Jakubczyk and W. Respondek Eds., Marcel Dekker, New York, pp. 463-557, 1997.

${ }^{27}$ Crouch, P. E., 'Spacecraft attitude control and stabilisation: Applications of geometric control theory to rigid body models” IEEE. Transactions on automatic control, vol. 29, No. 4, 1984. 
${ }^{28}$ Leonard, N. E., Krishnaprasad, P. S., 'Motion control of drift-free, left-invariant systems on Lie groups' IEEE. Transactions on automatic control, vol. 40, No. 9, pp. 1539-1554, 1995.

${ }^{29}$ Spindler, K., 'Optimal Attitude Control of a Rigid Body'. Applied Mathematics and optimisation, vol 34, pp. 79-90, 1996.

${ }^{30}$ Murray, R. M., Sastry, S. S., 'Nonholonomic motion planning: Steering using sinusoids'. IEEE Transactions on Automatic Control, Vol. 38, No.5, pp. 700-716, 1993.

${ }^{31}$ Ruskeepaa, H., 'Mathematica Navigator: Mathematics, Statistics and Graphics', Academic Press, Third Edition, 2009.

${ }^{32}$ Luo , W., Chu, Y., Ling; K. 'H1 Inverse optimal attitude tracking control of rigid spacecraft', AIAA Journal of Guidance, Control and Dynamics, vol. 28, no.3, pp. 481-493, 2005.

${ }^{33}$ Khalil, H.K., 'Nonlinear Systems', 3rd ed., Prentice Hall, Englewood Cliffs, NJ, pp.126-132, 2002. 